\title{
Optimization Strategies for Integration Pattern Compositions
}

\author{
Daniel Ritter, Norman May \\ SAP SE \\ Walldorf (Baden), Germany \\ \{firstname.lastname\}@sap.com
}

\author{
Fredrik Nordvall Forsberg \\ University of Strathclyde \\ Glasgow, Scotland \\ fredrik.nordvall-forsberg@strath.ac.uk
}

\author{
Stefanie Rinderle-Ma \\ University of Vienna \\ Vienna, Austria
}

stefanie.rinderle-ma@univie.ac.at

\begin{abstract}
Enterprise Application Integration is the centerpiece of current on-premise, cloud and device integration scenarios. We describe optimization strategies that help reduce the model complexity, and improve the process execution using design time techniques. In order to achieve this, we formalize compositions of Enterprise Integration Patterns based on their characteristics, and propose a realization of optimization strategies using graph rewriting. The framework is successfully evaluated on a real-world catalog of pattern compositions, containing over 900 integration scenarios.
\end{abstract}

\section{CCS CONCEPTS}

- Applied computing $\rightarrow$ Enterprise application integration; - Information systems $\rightarrow$ Data exchange;

\section{KEYWORDS}

Enterprise Application Integration, Enterprise Integration Patterns, Optimization Strategies.

\section{ACM Reference Format:}

Daniel Ritter, Norman May, Fredrik Nordvall Forsberg, and Stefanie RinderleMa. 2018. Optimization Strategies for Integration Pattern Compositions. In DEBS '18: The 12th ACM International Conference on Distributed and Eventbased Systems, Fune 25-29, 2018, Hamilton, New Zealand. ACM, New York, NY, USA, 12 pages. https://doi.org/10.1145/3210284.3210295

\section{INTRODUCTION}

Enterprise Application Integration (EAI) is the centerpiece of current IT infrastructure and integration scenarios, and essentially amounts to composing Enterprise Integration Patterns (EIPs) from a catalog comprising the original patterns [23] and recent additions [37, 40]. This can result in complex models that are often vendor-specific, informal and ad-hoc [37]; optimizing such integration processes is desirable, but hard. In most cases this is further complicated by data aspects being absent in the model. As a concrete motivation for a formal framework for data-aware integration process optimization, consider the following example: many organizations have started to connect their on-premise applications such as Customer Relationship Management (CRM) systems with cloud applications such as SAP Cloud for Customer (COD) using

Permission to make digital or hard copies of all or part of this work for personal or classroom use is granted without fee provided that copies are not made or distributed for profit or commercial advantage and that copies bear this notice and the full citation on the first page. Copyrights for components of this work owned by others than ACM must be honored. Abstracting with credit is permitted. To copy otherwise, or republish, to post on servers or to redistribute to lists, requires prior specific permission and/or a fee. Request permissions from permissions@acm.org.

DEBS '18, June 25-29, 2018, Hamilton, New Zealand

(C) 2018 Association for Computing Machinery.

ACM ISBN 978-1-4503-5782-1/18/06 ..\$15.00

https://doi.org/10.1145/3210284.3210295

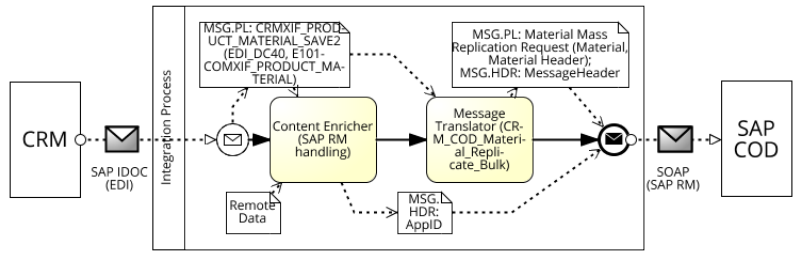

Figure 1: SAP Hybris Cloud Replicate Material from SAP Business Suite (a "hybrid integration" scenario).

integration processes similar to the one shown in Fig. 1. A CRM Material is sent from the CRM system via EDI (more precisely SAP IDOC transport protocol) to an integration process running on SAP Cloud Platform Integration (SAP CPI) ${ }^{1}$. The integration process enriches the message header (MSG.HDR) with additional information based on a document number for reliable messaging (i.e., AppID), which allows redelivery of the message in an exactly-once service quality [40]. The IDOC structure is then mapped to the COD service description and sent to the COD receiver. Already in this simple scenario an obvious improvement can be applied: the data-independent Content Enricher and Message Translator patterns [23] could be executed in parallel. This insight would perhaps not be obvious without the data flow in the model, and leads to questions such as the following: "are there other optimizations that could also be applied, and how can the modeling be supported by this?". Currently these questions cannot be answered, since approaches for verification and static analysis of "realistic data-aware" business and integration processes are missing, as recent surveys on event data [1, 16], workflow management [27], and in particular application integration [37] report. Hence, this work aims to fill this gap, based on the following research questions:

RQ1 What are relevant optimization techniques for EAI pattern compositions?

RQ2 How can pattern compositions be suitably formalized for optimizations?

RQ3 How can optimization strategies be formally defined?

In this work, we develop a verification and static analysis framework for applying and reasoning about optimizations of data-aware integration patterns. Our main contribution is a graph-based representation of integration patterns, so that optimizations can be realized as graph rewriting rules. To show the feasibility of our framework, we analyzed its use on a catalog of over 900 real-world

\footnotetext{
${ }^{1}$ SAP CPI, visited May 2018: https://api.sap.com/shell/discover. The pattern compositions in this catalog are represented in a BPMN model (e.g., [40]), and thus we subsequently represent our examples in this way - this is also more expressive in terms of message and data representation than the EIP icon notation [23].
} 
Table 1: Optimizations in related domains - horizontal search

\begin{tabular}{|c|c|c|c|c|}
\hline Keyword & hits & selected & Selection criteria & Selected Papers \\
\hline Business Process Optimization & 159 & 3 & data-aware processes & survey [44], optimization patterns $[31,32]$ \\
\hline Workflow Optimization & 396 & 6 & data-aware processes & $\begin{array}{l}\text { instance scheduling }[2,7,43] \text {, scheduling and partitioning for in- } \\
\text { teraction [3], scheduling and placement [6], operator merge [22] }\end{array}$ \\
\hline Data Integration Optimization & 61 & 2 & $\begin{array}{l}\text { data-aware processes optimization, } \\
\text { (no schema-matching) }\end{array}$ & $\begin{array}{l}\text { instance scheduling, parallelization [46], ordering, materialization, } \\
\text { arguments, algebraic [19] }\end{array}$ \\
\hline Added & $\mathrm{n} / \mathrm{a}$ & 8 & expert knowledge & $\begin{array}{l}\text { business process }[45] \text {, workflow survey }[27,28] \text {, data integration } \\
{[12] \text {, distributed applications }[8,9] \text {, EAI }[35,36]}\end{array}$ \\
\hline Removed & - & 1 & & classification only [44] \\
\hline Overall & 616 & 18 & & \\
\hline
\end{tabular}

integration scenarios. With our approach, we can show that $81 \%$ of the original scenarios from 2015 and still up to $52 \%$ of the current SAP CPI content from 2017 could be improved through a parallelization of scenario parts. We stress that we use the word "optimization" here in the sense of, e.g., an optimizing compiler: a process which iteratively improves compositions, but gives no guarantee of optimality. Due to brevity, we focus on the common EAI optimization objectives [23]: message throughput (on experimental runtime benchmarks), pattern processing latency (on an abstract cost model), and also runtime independent model complexity [42] from the process modeling domain. Furthermore, we concentrate on pattern compositions within one integration process (not to or within message endpoints).

Methodology. Overall, we follow the design science research approach from Peffers et al. [33] to systematically analyze the state-ofthe-art. At first, we collect optimization techniques as optimization strategies from related domains (answering RQ1) by conducting a horizontal literature search based on Kitchenham [26]. We opt for a high-level representation of pattern compositions as control flow graphs [4] (where the nodes represent extended EIPs) with communication contracts. This allows our representation to specify data and throughput aspects - which BPMN-based models cannot [40] - whilst still modeling the composition logic of the patterns, rather than their internal logic as in Petri Net-based models [17] (answering RQ2). We then formalize the optimization strategies based on the contract graphs using graph rewriting techniques (answering RQ3), and evaluate them on real-world pattern compositions.

Outline. In Sect. 2, we collect optimization techniques, identify requirements for optimizing EIP compositions, and classify the optimization strategies and optimization objectives (i.e., modeling complexity, processing latency, message throughput). Our formal model of pattern compositions is introduced in Sect. 3. Optimization strategies are then formalized in Sect. 4, and evaluated on case studies based on the objectives in Sect. 5. We discuss related work in Sect. 6 and conclude in Sect. 7.

\section{STATIC OPTIMIZATION STRATEGIES}

In this section we survey recent attempts to optimize composed EIPs, in order to motivate the need to formalize their semantics. As a result, we derive three so far unexplored prerequisites R1-R3 for optimizing compositions of EIPs.

\subsection{Identifying optimization strategies}

Since a formalization of the EAI foundations in the form of integration patterns for static optimization of "data-aware" pattern processing is missing [37], we conducted a horizontal literature search [26] to identify optimization techniques in related domains. For EAI, the domains of business processes, workflow management and data integration are of particular interest. The results of our analysis are summarized in Tab. 1. Out of the resulting 616 hits, we selected 18 papers according to the search criteria "data-aware processes", and excluded work on unrelated aspects. Table 2 lists the optimization techniques, already mapped to EAI, and skipping those techniques that do not provide solutions for our optimization objectives or within an integration process. This resulted in the seven papers cited in the table. The mapping of techniques from related domains to EAI was done by for instance taking the idea of projection push-downs $[11,19,22,31,45]$ and deriving the early-filter or early-mapping technique in EAI. We categorized the techniques according to their impact (e.g., structural or process, data-flow) in context of the objectives for which they provide solutions.

In the following subsections, we now briefly discuss the optimization strategies listed in Tab. 2, in order to derive prerequisites needed for optimizing compositions of EIPs. To relate to their practical relevance and coverage so far (in the form of evaluations on "real-world" integration scenarios), we also discuss existing "dataaware" message processing solutions for each group of strategies.

\subsection{Process Simplification}

We grouped together all techniques whose main goal is reducing model complexity (i.e., number of patterns) under the heading of process simplification. The cost reduction of these techniques can be measured by pattern processing time (latency, i.e., time required per operation) and model complexity metrics [42]. Process simplification can be achieved by removing redundant patterns like Redundant Subprocess Removal (e.g., remove one of two identical sub-flows), Combine Sibling Patterns (e.g., remove one of two identical patterns), or Unnecessary Conditional Fork (e.g., remove redundant branching). As far as we know, the only practical study of combining sibling patterns can be found in Ritter et al. [36], showing moderate throughput improvements. The simplifications requires a formalization of patterns as a control graph structure (R1), which helps to identify and deal with the structural change representation. Previous work targeting process simplification include Böhm et al. [11] and Habib, Anjum and Rana [22], who use 
Table 2: Optimization Strategies in context of the objectives

\begin{tabular}{|c|c|c|c|c|c|}
\hline Strategy & Optimization & Throughput & Latency & Complexity & Practical Studies \\
\hline OS-1: Process & Redundant Sub-process Removal [11] & $+/-$ & + & + & - \\
\hline \multirow{2}{*}{ Simplification } & Combine Sibling Patterns $[11,22]$ & $+/-$ & + & + & $([36])$ \\
\hline & Unnecessary conditional fork $[11,45]$ & $(+)$ & + & + & - \\
\hline \multirow[t]{5}{*}{ OS-2: Data Reduction } & Early-Filter $[11,19,22,31,45]$ & + & $+/-$ & $+/-$ & {$[36]$} \\
\hline & Early-Mapping [11, 19, 22] & + & $+/-$ & $+/-$ & {$[36,39]$} \\
\hline & Early-Aggregation $[11,19,22]$ & + & $+/-$ & $+/-$ & [39] \\
\hline & Claim Check $[11,19]$ & + & $+/-$ & - & - \\
\hline & Early-Split [36] & + & $+/-$ & - & {$[36,39]$} \\
\hline \multirow[t]{2}{*}{ OS-3: Parallelization } & Sequence to parallel $[11,31,45,46]$ & + & $+/-$ & - & {$[35,36]$} \\
\hline & Merge parallel sub-processes $[11,31,45,46]$ & $+/-$ & + & + & [36] \\
\hline
\end{tabular}

evolutionary search approaches on workflow graphs, and Vrhovnik et al. [45], who use a rule formalization on query graphs.

\subsection{Data Reduction}

The reduction of data can be facilitated by pattern push-down optimizations of message-element-cardinality-reducing patterns, which we call Early-Filter (for data; e.g., remove elements from the message content), Early-Mapping (e.g., apply message transformations), as well as message-reducing optimization patterns like Early-Filter (for messages; e.g., remove messages), Early-Aggregation (e.g., combine multiple messages to fewer ones), Early-Claim Check (e.g., store content and claim later without passing it through the pipeline), and Early-Split (e.g., cut one large message into several smaller ones). Measuring data reduction requires a cost model based on the characteristics of the patterns, as well as the data and element cardinalities. For example, the practical realizations for multimedia [39] and hardware streaming [36] show improvements especially for early-filter, split and aggregation, as well as moderate improvements for earlymapping. This requires a formalization that is able to represent data or element flow (R2). Data reduction optimizations target message throughput improvements (i.e., processed messages per time unit), however, some have a negative impact on the model complexity. Previous work on data reduction include Getta [19], who targets optimization techniques on relational algebra expressions, and Niedermann, Radeschütz and Mitschang [31], who define optimizations algorithmically for a graph-based model.

\subsection{Parallelization}

Parallelization of processes can be facilitated through transformations such as Sequence to Parallel (e.g., duplicate pattern or sequence of pattern processing), or, if not beneficial, reverted, e.g., by Merge Parallel. For example, good practical results have been shown for vectorization [35] and hardware parallelization [36]. Therefore, again, a control graph structure (R1) is required. Although the main focus of parallelization is message throughput, heterogeneous variants also improve latency. In both cases, parallelization requires additional patterns, which negatively impacts the model complexity. The opposite optimization of merging parallel processes mainly improves the model complexity and latency. Previous work on pattern parallelization include Zhang et al. [46], who defines a service composition model, to which algorithmically defined optimizations are applied.

\subsection{Discussion}

Due to our objectives and our focus on optimizations within a process, the collection of optimizations in Tab. 2 is not complete. For instance, we have not treated pattern placement optimizations (pushing patterns to message endpoints, i.e., sender and receiver applications), or optimizations that reduce interaction (helping to stabilize the process). Besides control flow (as used in most of the related domains), a suitable formalization must be able to represent the control graph structure (R1) (including reachability and connectedness properties) and the data element flow (R2) between patterns (not within a pattern). Furthermore, the formalization must allow verification of correctness (R3) on a pattern-compositional level (i.e., each optimization produces a correct pattern composition), taking the inter-pattern data exchange semantics into account. In contrast to the related work, we define a novel data-aspect representation of the extended EIPs and guarantee correctness.

\section{GRAPH-BASED PATTERN COMPOSITIONS}

In this section, we introduce our formalization of pattern compositions, and an abstract cost model for them. Such a formalization is needed in order to talk about optimizations rigorously.

\subsection{Integration Pattern Graphs}

Summarizing the requirements $\mathbf{R} \mathbf{1}-\mathbf{R} \mathbf{3}$ collected in the previous section, a suitable formalization of integration patterns is graphbased, can represent the data element flow, and allows correctness checking. Hence, we define an Integration Pattern Typed Graph (IPTG) as an extended control flow graph [4] as follows. Let us first fix some notation: a directed graph is given by a set of nodes $P$ and a set of edges $E \subseteq P \times P$. For a node $p \in P$, we write $\bullet p=$ $\left\{p^{\prime} \in P \mid\left(p^{\prime}, p\right) \in E\right\}$ for the set of direct predecessors of $p$, and $p \bullet=$ $\left\{p^{\prime \prime} \in P \mid\left(p, p^{\prime \prime}\right) \in E\right\}$ for the set of direct successors of $p$.

Definition 3.1. An integration pattern typed graph (IPTG) is a directed graph with set of nodes $P$ and set of edges $E \subseteq P \times P$, together with a function type $: P \rightarrow T$, where $T=\{$ start, end, message processor, fork, structural join, condition, merge, external call $\}$. An $\operatorname{IPTG}(P, E$, type $)$ is correct if

- $\exists p_{1}, p_{2} \in \mathrm{P}$ with type $\left(p_{1}\right)=$ start and $\operatorname{type}\left(p_{2}\right)=$ end;

- if type $(p) \in\{$ fork, condition $\}$ then $|\bullet p|=1$ and $|p \bullet|=n$, and if type $(p)=$ join then $|\bullet p|=n$ and $|p \bullet|=1$;

- if type $(p) \in$ \{message processor, merge $\}$ then $|\bullet p|=1$ and $|p \bullet|=1$ 
- if $\operatorname{type}(p) \in\{$ external call $\}$ then $|\bullet p|=1$ and $|p \bullet|=2$;

- The graph $(P, E)$ is connected and acyclic.

In the definition, we think of $P$ as a set of extended EIPs that are connected by message channels in $E$, as in a pipes and filter architecture. The function type records what type of pattern each node represents. The first correctness condition says that an integration pattern has at least one source and one target, while the next three states the cardinality of the involved patterns coincide with the in- and out-degrees of the nodes in the graph representing them. The last condition states that the graph represents one integration pattern, not multiple unrelated ones, and that messages do not loop back to previous patterns.

To represent the data flow, i.e., the basis for the optimizations, the control flow has to be enhanced with (a) the data that is processed by each pattern, and (b) the data exchanged between the patterns in the composition. The data processed by each pattern (a) is described as a set of pattern characteristics, formally defined as follows:

Definition 3.2. A pattern characteristic assignment for an IPTG ( $P, E$, type $)$ is a function char $: P \rightarrow 2^{P C}$, assigning to each pattern a subset of the set

$$
\begin{aligned}
P C= & (\{\mathrm{MC}\} \times \mathbb{N} \times \mathbb{N}) \cup \\
& (\{\mathrm{ACC}\} \times\{\text { ro, rw }\}) \cup \\
& (\{\mathrm{MG}\} \times \mathbb{B}) \cup \\
& \left(\{\mathrm{CND}\} \times 2^{\mathrm{BExp}}\right),
\end{aligned}
$$

where $\mathbb{B}$ is the set of Booleans, BExp the set of Boolean expressions, and $\mathrm{MC}, \mathrm{CHG}, \mathrm{MG}, \mathrm{CND}$ some distinct symbols.

The property and value domains in the definition are based on the pattern descriptions in [23,37], and could be extended if further analysis required it. We briefly explain the intuition behind the characteristics: the characteristic $(\mathrm{MC}, n, k)$ represents a message cardinality of $n: k,(\mathrm{ACC}, x)$ the message access, depending on if $x$ is read-only ro or read-write rw, and the characteristic (MG, $y$ ) represents whether the pattern is message generating depending on the Boolean $y$. Finally (CND, $X$ ) represents the conditions for the pattern collected in the set of Boolean expressions $X$.

Example 3.3. The characteristics of a content-based router $C B R$ is $\operatorname{char}(C B R)=\left\{(\mathrm{MC}, 1: 1),(\mathrm{ACC}, \mathrm{ro}),(\mathrm{MG}\right.$, false $),\left(\mathrm{CND},\left\{\right.\right.$ cnd $_{1}, \ldots$, $\left.\left.\left.c n d_{n-1}\right\}\right)\right\}$, because of the workflow of the router: it receives exactly one message, then evaluates up to $n-1$ routing conditions $c n d_{1}$ up to $c n d_{n-1}$ (one for each outgoing channel), until a condition matches. The original message is then rerouted read-only (in other words, the router is not message generating) on the selected output channel, or forwarded to the default channel, if no condition matches.

The data exchange between the patterns (b) is based on input and output contracts (similar to data parallelization contracts in [5]). These contracts specify how the data is exchanged in terms of required message properties of a pattern during the data exchange, formally defined as follows:

Definition 3.4. A pattern contract assignment for an IPTG $(P, E$, type $)$ is a function contr $: P \rightarrow 2^{C P T} \times 2^{E L}$, assigning to each pattern a subset of the set

$$
C P T=\{\text { signed, encrypted, encoded }\} \times\{\text { yes, no, any }\}
$$

and a subset of the set

$$
E L=M S \times 2^{D}
$$

where $M S=\{\mathrm{HDR}, \mathrm{PL}, \mathrm{ATTCH}\}$, and $D$ is a set of data elements (the concrete elements of $D$ are not important, and will vary with the application domain).

Each pattern will have an inbound and an outbound pattern contract, describing the format of the data it is able to receive and send respectively - the role of pattern contracts is to make sure that adjacent inbound and outbound contracts match. The set $C P T$ in a contract represents integration concepts, while the set $E L$ represents data elements and the structure of the message: its headers (HDR, $H$ ), its payload (PL, $Y$ ) and its attachments (ATTCH, $A$ ).

Example 3.5. A content-based router is not able to process encrypted messages. Recall that its pattern characteristics included a collection of routing conditions: these might require read-only access to message elements such as certain headers $h_{1}$ or payload elements $e_{1}, e_{2}$. Hence the input contract for a router mentioning these message elements is

inContr $(C B R)=\left(\{(\right.$ encrypted, no $\left.)\},\left\{\left(H D R,\left\{h_{1}\right\}\right),\left(\mathrm{PL},\left\{e_{1}, e_{2}\right\}\right)\right\}\right)$.

Since the router forwards the original message, the output contract is the same as the input contract.

Definition 3.6. Let $(C, E) \in 2^{C P T} \times 2^{E L}$ be a pattern contract, and $X \subseteq 2^{C P T} \times 2^{E L}$ a set of pattern contracts. Write $X_{C P T}=$ $\left\{C^{\prime} \mid\left(\exists E^{\prime}\right)\left(C^{\prime}, E^{\prime}\right) \in X\right\}$ and $X_{E L}=\left\{E^{\prime} \mid\left(\exists C^{\prime}\right)\left(C^{\prime}, E^{\prime}\right) \in X\right\}$. We say that $(C, E)$ matches $X$, in symbols match $((C, E), X)$, if the following condition holds:

$$
\begin{aligned}
& (\forall(p, x) \in C)\left(x=\text { any } \vee\left(\forall C^{\prime} \in X_{C P T}\right)\left(\exists\left(p^{\prime}, y\right) \in C^{\prime}\right)\right. \\
& \left.\left(p=p^{\prime} \wedge(y=\text { any } \vee y=x)\right)\right) \wedge \\
& (\forall(m, Z) \in E)\left(Z \subseteq \bigcup_{\left(m, Z^{\prime}\right) \in \cup X_{E L}} Z^{\prime}\right)
\end{aligned}
$$

We are interested in an inbound contract $K_{\text {in }}$ matching the outbound contracts $K_{1}, \ldots, K_{n}$ of its predecessors. In words, this is the case if (i) for all integration concepts that are important to $K_{\text {in }}$, all contracts $K_{i}$ either agree, or at least one of $K_{\text {in }}$ or $K_{i}$ accepts any value; and (ii) together, $K_{1}, \ldots, K_{n}$ supply all the message elements that $K_{\text {in }}$ needs.

Since pattern contracts can refer to arbitrary message elements, a formalization of an integration pattern can be quite precise. On the other hand, unless care is taken, the formalization can easily become specific to a particular pattern composition. In practice, it is often possible to restrict attention to a small number of important message elements (see Example 3.8 below), which makes the formalization manageable.

Putting everything together, we formalize pattern compositions as integration pattern typed graphs with pattern characteristics and inbound and outbound pattern contracts for each pattern:

Definition 3.7. An integration pattern contract graph (IPCG) is a tuple

$$
\text { ( } P, \text { E, type, char, inContr, outContr) }
$$

where $(P, E$, type $)$ is an IPTG, char $: P \rightarrow 2^{P C}$ is a pattern characteristics assignment, and inContr, outContr $: P \rightarrow 2^{C P T} \times 2^{E L}$ are 
pattern contract assignments, called the inbound and outbound contract assignment respectively. It is correct, if the underlying IPTG $(P, E$, type $)$ is correct, and inbound contracts matches the outbound contracts of the patterns' predecessors, i.e.

$(\forall p)\left(p=\operatorname{start} \vee \operatorname{match}\left(\operatorname{inContr}(p),\left\{\operatorname{outContr}\left(p^{\prime}\right) \mid p^{\prime} \in \bullet p\right\}\right)\right)$.

Two IPCGs are isomorphic if there is a bijective function between their patterns that preserves edges, types, characteristics and contracts.

Example 3.8. Figures 2(a) and 2(b) show IPCGs representing an excerpt of the motivating example from the introduction. Figure 2(a) represents the IPCG of the original scenario with a focus on the contracts, and Fig. 2(b) denotes an already improved composition showing the characteristics and giving an indication on the pattern latency. In Fig. 2(a), the input contract inContr(CE) of the content enricher pattern $C E$ requires a non-encrypted message and a payload element DOCNUM. The content enricher makes a query to get an application ID AppID from an external system, and appends it to the message header. Hence the output contract outContr $(C E)$ contains (HDR, $\{$ AppID $\}$ ). The content enricher then emits a message that is not encrypted or signed. A subsequent message translator $M T$ requires the same message payload, but does not care about the appended header. It adds another payload RcvID to the message. Comparing inbound and outbound pattern contracts for adjacent patterns, we see that this is a correct IPCG.

One improvement of this composition is depicted in Fig. 2(b), where the independent patterns $C E$ and $M T$ have been parallelized. To achieve this, a read-only structural fork with channel cardinality $1: n$ in the form of a multicast $M C$ has been added. The inbound and outbound contracts of $M C$ are adapted to fit into the composition. After the concurrent execution of $C E$ and $M T$, a join router $J R$ brings the messages back together again and feeds the result into an aggregator $A G G$ that restores the format that $A D P T_{r}$ expects. We see that the resulting IPCG is still correct, so this would be a sound optimization.

\subsection{Abstract Cost Model}

In order to decide if an optimization is an improvement or not, we want to associate abstract costs to integration patterns. We do this on the pattern level, similar to the work on data integration operators [10]. The cost of the overall integration pattern can then be computed as the sum of the cost of its constituent patterns. Costs are considered parameterized by the cardinality of data inputs $\left|d_{\mathrm{in}_{i}}\right|$ ( $1 \leq i \leq n$, if the pattern has in-degree $n$ ), data outputs $\left|d_{\text {out }_{j}}\right|$ $(1 \leq j \leq m$, if the pattern has out-degree $m)$, and external resource data sets $\left|d_{r}\right|$. The costs can also refer to the pattern characteristics.

Definition 3.9. A cost assignment for an IPCG (P, E, type, char, inContr, outContr) is an function $\operatorname{cost}(p): \mathbb{N}^{n} \times \mathbb{N}^{k} \times \mathbb{N}^{r} \rightarrow \mathbb{Q}$ for each $p \in P$, where $p$ has in-degree $n$, out-degree $k$ and $r$ external connections. The cost $\operatorname{cost}(G): \mathbb{N}^{N} \times \mathbb{N}^{K} \times \mathbb{N}^{R} \rightarrow \mathbb{Q}$ of an IPCG pattern graph $G=(P, E, t y p e, p c, i c, o c)$ with a cost assignment, where $N$ is the sum of the in-degrees of its patterns, $K$ the sum of their out-degrees, and $R$ the sum of their external connections, is defined to be the sum of the costs of its constituent patterns:

$$
\operatorname{cost}(G)\left(d_{\text {in }}, d_{\text {out }}, d_{r}\right)=\sum_{p \in P} \operatorname{cost}(p)\left(\left|d_{\text {in }}(p)\right|,\left|d_{\text {out }}(p)\right|,\left|d_{r}(p)\right|\right)
$$

Table 3: Abstract costs of relevant patterns

\begin{tabular}{|c|c|c|}
\hline Pattern $p$ & Abstract Cost $\cos t(p)$ & Factors \\
\hline Content-based Router [23] & $\frac{\sum_{i=0}^{n-1}\left|d_{\mathrm{in}, i}\right|}{2}$ & $\begin{array}{l}n=\# \text { channel conditions, half of them } \\
\text { evaluated in average }\end{array}$ \\
\hline Message Filter [23] & $\left|d_{\text {in }}\right|$ & input data condition $\left|d_{\text {in }}\right|$ \\
\hline Aggregator [23] & $2 \times\left|d_{\text {in }}\right|+\frac{\left|d_{\text {in }}\right|+\left|d_{r}\right|}{\operatorname{avg}(\text { len }(\text { seq }))}$ & $\begin{array}{l}\text { correlation, and completion condi- } \\
\text { tions }\left|d_{\text {in }}\right| \text {, aggregation function } \\
\frac{\left|d_{\text {in }}\right|+\left|d_{r}\right|}{a v g(l e n(s e q))} \text { and length of a se- } \\
\text { quence length(seq) }>=2 \text {, and (trans- } \\
\text { acted) resource } d_{r} \\
\text { resource insert and get }\left|d_{r}\right|\end{array}$ \\
\hline Splitter [23] & $\left|d_{\text {out }}\right|$ & - \\
\hline Multicast, JoinRouter [37] & $\sum_{i=0}^{n} \operatorname{cost}\left(\right.$ procunit $\left._{i}\right)$ & $\begin{array}{l}\text { costs of processing units } \\
\operatorname{cost}\left(\text { procunit }_{i}\right) \text {, e.g., threading } \\
\text { in software, for } n \text { channels }\end{array}$ \\
\hline Content Filter [23] & $\left|d_{\text {out }}\right|$ & output data creation $\left|d_{\text {out }}\right|$ \\
\hline Mapping [23] & $\left|d_{\text {in }}\right|+\left|d_{\text {out }}\right|$ & $\begin{array}{l}\text { output data creation }\left|d_{\text {out }}\right| \text { from in- } \\
\text { put data }\left|d_{\text {in }}\right|\end{array}$ \\
\hline Content Enricher [23] & $\left|d_{\text {in }}\right|+\left|d_{r}\right|+\left|d_{\text {out }}\right|$ & $\begin{array}{l}\text { request message creation on }\left|d_{\text {in }}\right| \text {, } \\
\text { resource query }\left|d_{r}\right| \text {, response data } \\
\text { enrich }\left|d_{\text {out }}\right|\end{array}$ \\
\hline External Call [37] & $\left|d_{\text {out }}\right|+\left|d_{\text {in }}\right|$ & request $\left|d_{\text {out }}\right|$ and reply data $\left|d_{\text {in }}\right|$ \\
\hline Receive [23] & $\left|d_{\text {in }}\right|$ & input data $\left|d_{\text {in }}\right|$ \\
\hline Send [23] & $\left|d_{\text {out }}\right|$ & output data $\left|d_{\text {out }}\right|$ \\
\hline
\end{tabular}

where we suggestively have written $\left|d_{\text {in }}(p)\right|$ for the projection from the tuple $d_{\text {in }}$ corresponding to $p$, similarly for $\left|d_{\text {out }}(p)\right|$ and $\left|d_{r}(p)\right|$.

We have defined the abstract costs of the patterns discussed in this work in Tab. 3 - these will be used in the evaluation in Sect. 5. We now explain the reasoning behind them. Routing patterns such as content based routers, message filters and aggregators mostly operate on the input message, and thus have an abstract cost related to its element cardinality $\left|d_{\text {in }}\right|$. For example, the abstract cost of the content-based router is $\cos t(C B R)=\frac{\sum_{i=0}^{n-1}\left|d_{\mathrm{in}, i}\right|}{2}$, since it evaluates on average $\frac{n-1}{2}$ routing conditions on the input message. More complex routing patterns such as aggregators evaluate correlation and completion conditions, as well as an aggregation function on the input message, and also on sequences of messages of a certain length from an external resource. Hence the cost of an aggregator is $\operatorname{cost}(A G G)=2 \times\left|d_{\text {in }}\right|+\frac{\left|d_{\text {in }}\right|+\left|d_{r}\right|}{\operatorname{avg}(\text { len }(\text { seq }))}$. In contrast, message transformation patterns like content filters and enrichers mainly construct an output message, hence their costs are determined by the output cardinality $\left|d_{\text {out }}\right|$. For example, content enrichers create a request message from the input message with cost $\left|d_{\text {in }}\right|$, conducts an optional resource query $\left|d_{r}\right|$, and creates and enriches the response with cost $\left|d_{\text {out }}\right|$. Finally, the cost of message creation patterns such as external calls, receivers, and senders arise from costs for transport, protocol handling, and format conversion, as well as decompression. Hence the cost depends on the element cardinalities of input and output messages $\left|d_{\text {in }}\right|,\left|d_{\text {out }}\right|$.

Example 3.10. We return to the claimed improved composition in Example 3.8. The latency of the composition $G_{1}$ in Fig. 2(a), calculated from the constituent pattern latencies, is $\operatorname{cost}\left(G_{1}\right)=t_{C E}+t_{M T}$. The latency improvement potential given by switching to the composition $G_{2}$ in Fig. 2(b) is given by $\operatorname{cost}\left(G_{2}\right)=\max \left(t_{C E}, t_{M T}\right)+$ $t_{M C}+t_{J R}+t_{A G G}$. Obviously it is only beneficial to switch if $\operatorname{cost}\left(G_{2}\right)<\operatorname{cost}\left(G_{1}\right)$, and this condition depends on the concrete values involved. At the same time, the model complexity increases by three nodes and edges. 


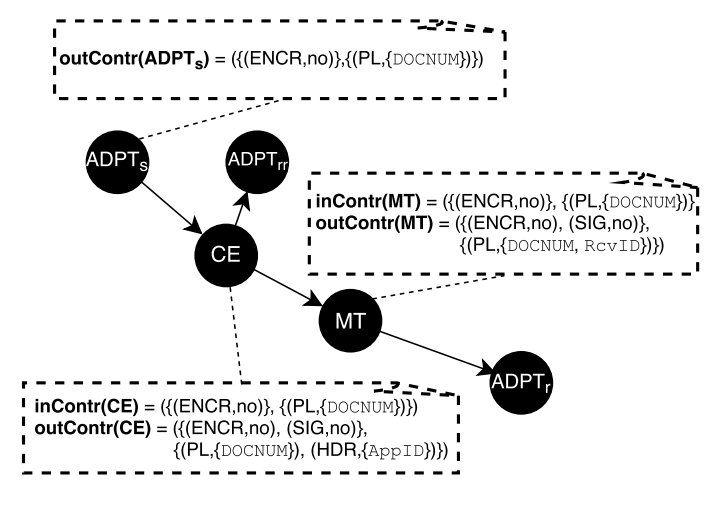

(a) IPCG from the motivating example

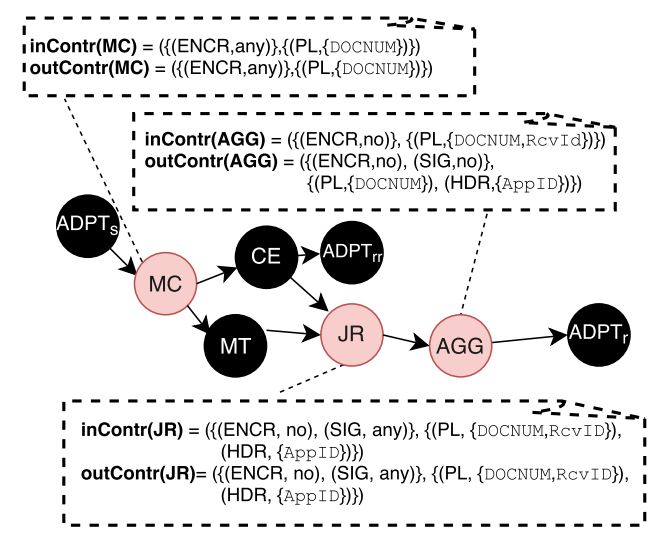

(b) IPCG after "sequence to parallel" optimization

Figure 2: An IPCG of an excerpt of the motivating example.

\section{OPTIMIZATION STRATEGY REALIZATION}

In this section we formally define the optimizations from the different strategies identified in Tab. 2 in the form of a rule-based graph rewriting system. This gives a formal framework in which different optimizations can be compared. We begin by describing the graph rewriting framework, and subsequently apply it to define the optimizations.

\subsection{Graph Rewriting}

Graph rewriting provides a visual framework for transforming graphs in a rule-based fashion. A graph rewriting rule is given by two embeddings of graphs $L \hookleftarrow K \hookrightarrow R$, where $L$ represents the left hand side of the rewrite rule, $R$ the right hand side, and $K$ their intersection (the parts of the graph that should be preserved by the rule). A rewrite rule can be applied to a graph $G$ after a match of $L$ in $G$ has been given as an embedding $L \hookrightarrow G$; this replaces the match of $L$ in $G$ by $R$. The application of a rule is potentially non-deterministic: several distinct matches can be possible [14] Visually, we represent a rewrite rule by a left hand side and a right hand side graph colored green and red: green parts are shared and represent $K$, while the red parts are to be deleted in the left hand side, and inserted in the right hand side respectively. For instance, the following rewrite rule moves the node $P_{1}$ past a fork by making a copy in each branch, changing its label from $c$ to $c^{\prime}$ in the process:

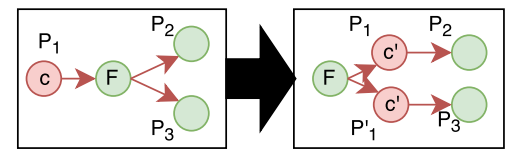

Formally, the rewritten graph is constructed using a double-pushout (DPO) [15] from category theory. We use DPO rewriting since rule applications are side-effect free (e.g., no "dangling" edges) and local (i.e., all graph changes are described by the rules). We additionally use Habel and Plump's relabeling DPO extension [21] to facilitate the relabeling of nodes in partially labeled graphs. In Fig. 2, we showed contracts and characteristics in dashed boxes, but in the rules that follow, we will represent them as (schematic) labels inside the nodes for space reasons.
In addition, we also consider rewrite rules parameterized by graphs, where we draw the parameter graph as a cloud (see e.g., Fig. 3(a) for an example). A cloud represents any graph, sometimes with some side-conditions that are stated together with the rule. When looking for a match in a given graph $G$, it is of course sufficient to instantiate clouds with subgraphs of $G$ - this way, we can reduce the infinite number of rules that a parameterized rewrite rule represents to a finite number. Parameterized rewrite rules can formally be represented using substitution of hypergraphs [34] or by !-boxes in open graphs [25]. Since we describe optimization strategies as graph rewrite rules, we can be flexible with when and in what order we apply the strategies. We apply the rules repeatedly until a fixed point is reached, i.e., when no further changes are possible, making the process idempotent. Each rule application preserves IPCG correctness in the sense of Definition 3.7, because input contracts do not get more specific, and output contracts remain the same. Methodologically, the rules are specified by preconditions, change primitives, post-conditions and an optimization effect, where the pre- and post-conditions are implicit in the applicability and result of the rewriting rule.

\subsection{OS-1: Process Simplification}

We first consider the process simplification strategies from Sect. 2 OS-1 to OS-3 that mainly strive to reduce the model complexity and latency.

4.2.1 Redundant sub-process. This optimization removes redundant copies of the same sub-process within a process.

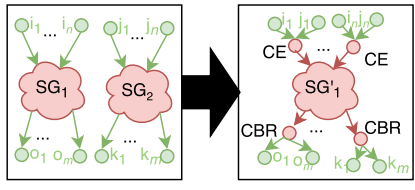

(a) Redundant sub-process

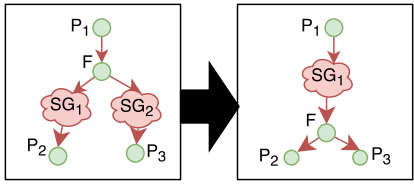

(b) Combine sibling patterns
Figure 3: Rules for redundant sub-process and combine sibling patterns. 
Change primitives: The rewriting is given by the rule in Fig. 3(a), where $S G 1$ and $S G 2$ are isomorphic pattern graphs with in-degree $n$ and out-degree $m$. In the right hand side of the rule, the $C E$ nodes add the context of the predecessor node to the message in the form of a content enricher pattern, and the $C B R$ nodes are content-based routers that route the message to the correct recipient based on the context introduced by $C E$. The graph $S G_{1}^{\prime}$ is the same as $S G_{1}$, but with the context introduced by $C E$ copied along everywhere.

Effect: The optimization is beneficial for model complexity when the isomorphic subgraphs contain more than $n+m$ nodes, where $n$ is the in-degree and $m$ the out-degree of the isomorphic subgraphs. The latency reduction is by the factor of subgraphs minus the latency introduced by the $n$ extra nodes $C E$ and $m$ extra nodes $C B R$.

4.2.2 Combine sibling patterns. Sibling patterns have the same parent node in the pattern graph (e.g., they follow a non-conditional forking pattern) with channel cardinality of 1:1. Combining them means that only one copy of a message is traveling through the graph instead of two - for this transformation to be correct in general, the siblings also need to be side-effect free, i.e., no external calls, although this is not captured by our correctness criteria.

Change primitives: The rule is given in Fig. 3(b), where $S G_{1}$ and $S G_{2}$ are isomorphic pattern graphs, and $F$ is a fork.

Effect: The model complexity and latency are reduced by the model complexity and latency of $S G_{2}$.

\subsection{OS-2: Data Reduction}

Now, we consider data reduction optimization strategies, which mainly target improvements of the message throughput (incl. reducing element cardinalities). These optimizations require that pattern input and output contracts are regularly updated with snapshots of element data sets $E L_{\text {in }}$ and $E L_{\text {out }}$ from live systems (cf. Sect. 3), e.g., from experimental measurements through benchmarks [38].

4.3.1 Early-Filter. A filter pattern can be moved to or inserted prior to some of its successors to reduce the data to be processed. The following types of filters have to be differentiated:

- A message filter removes messages with invalid or incomplete content. It can be used to prevent exceptional situations, and thus improves stability.

- A content filter removes elements from messages, thus reduces the amount of data passed to subsequent patterns.

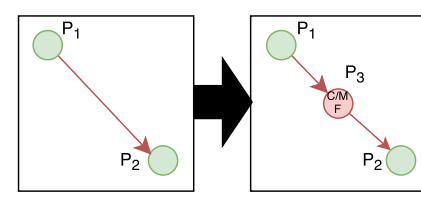

(a) Early Filter (b) Early Mapping

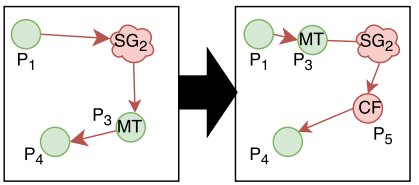

Figure 4: Rules for early-filter and early-mapping.

Change primitives: The rule is given in Fig. 4(a), where $P_{3}$ is either a content or message filter matching the output contracts of $P_{1}$ and the input contract of $P_{2}$, removing the data not used by $P_{2}$.
Effect: The message throughput increases by the ratio of the number of reduced data elements that are processed per second, unless limited by the throughput of the additional pattern.

4.3.2 Early-Mapping. A mapping that reduces the number of elements in a message can increase the message throughput.

Change primitives: The rule is given in Fig. 4(b), where $P_{3}$ is an element reducing message mapping compatible with both $S G_{2}, P_{4}$, and $P_{1}, S G_{2}$, and where $P_{4}$ does not modify the elements mentioned in the output contract of $P_{3}$. Furthermore $P_{5}$ is a content filter, which ensures that the input contract of $P_{4}$ is satisfied.

Effect: The message throughput for the subgraph subsequent to the mapping increases by the ratio of the number of unnecessary data elements processed.

\subsection{OS-3: Parallelization}

Parallelization optimization strategies increase message throughput. Again, these optimizations require experimentally measured message throughput statistics, e.g., from benchmarks [38].

4.4.1 Sequence to parallel. A bottleneck sub-sequence with channel cardinality 1:1 can also be handled by distributing its input and replicating its logic. The parallelization factor is the average message throughput of the predecessor and successor of the sequence divided by two, which denotes the improvement potential of the bottleneck sub-sequence. The goal is to not overachieve the mean of predecessor and successor throughput with the improvement to avoid iterative re-optimization. Hence the optimization is only executed, if the parallel sub-sequence reaches lower throughput than their minimum.

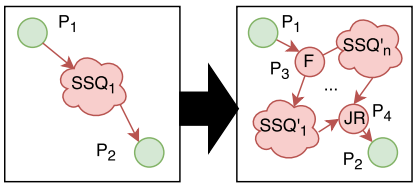

(a) Sequence to parallel

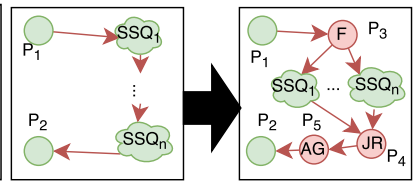

(b) Heterogeneous sequence to parallel
Figure 5: Rules for sequence to parallel variants.

Change primitives: The rule is given in Fig. 5(a), where $S S Q_{1}$ is a bottleneck sub-sequence, $P_{2}$ a fork node, $P_{3}$ a join router, and each $S S Q_{k}^{\prime}$ is a copy of $S S Q_{1}$, for $1 \leq k \leq n$. The parallelization factor $n$ is a parameter of the rule.

Effect: The message throughput improvement rate depends on the parallelization factor $n$, and the message throughput of the balancing fork and join router on the runtime. For a measured throughput $t$ of the bottleneck sub-sequences, the throughput can be improved to $n \times t \leq$ average of the sums of the predecessor and successor throughput, while limited by the upper boundary of the balancing fork or join router.

4.4.2 Heterogeneous Parallelization. A heterogeneous parallelization consists of parallel sub-sequences that are not isomorphic. In general, two subsequent patterns $P_{i}$ and $P_{j}$ can be parallelized, if the predecessor pattern of $P_{i}$ fulfills the input contract of $P_{j}, P_{i}$ behaves read-only with respect to the data element set of $P_{j}$, and the 


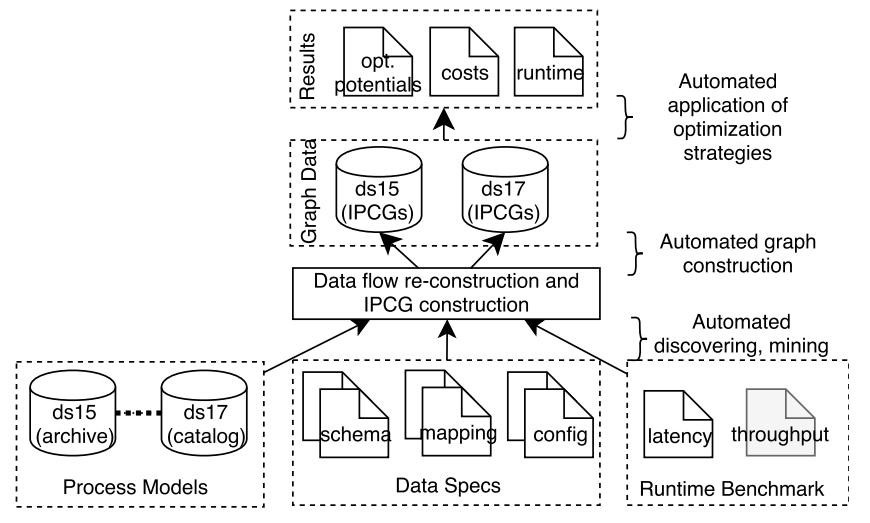

Figure 6: Pattern composition evaluation pipeline.

combined outbound contracts of $P_{i}$ and $P_{j}$ fulfill the input contract of the successor pattern of $P_{j}$.

Change primitives: The rule is given in Fig. 5(b), where the sequential sub-sequence parts $S S Q_{1}, . ., S S Q_{n}$ can be parallelized, $P_{3}$ is a parallel fork, $P_{4}$ is a join router, and $P_{5}$ is an aggregator that waits for messages from all sub-sequence part branches before emitting a combined message that fulfills the input contract of $P_{2}$.

Effect: Synchronization latency can be improved, but the model complexity increases by 3 . The latency improves from the sum of the sequential pattern latencies to the maximal latency of all sub-sequence parts plus the fork, join, and aggregator latencies.

\section{EVALUATION}

In this section, we apply the optimizations to integration processes from a commercial cloud integration system in a quantitative analysis, and exemplify the results by two real-world case studies.

\subsection{Quantitative Analysis}

We applied the optimization strategies OS-1-3 to 627 integration scenarios from the 2017 standard content of the SAP CPI (called ds17 below), and compared with 275 scenarios from 2015 (called ds15) Our goal is to show the applicability of our approach to real-world integration scenarios, as well as the scope and trade-offs of the optimization strategies. The comparison with a previous content version features a practical study on content evolution. To analyze the difference between different scenario domains, we grouped the scenarios into the following categories [37]: On-Premise to Cloud (OP2C), Cloud to Cloud (C2C), and Business to Business (B2B). Since hybrid integration scenarios such as OP2C target the extension or synchronization of business data objects, they are usually less complex. In contrast native cloud application scenarios such as $\mathrm{C} 2 \mathrm{C}$ or B2B mediate between several endpoints, and thus involve more complex integration logic [37]. The process catalog also contained a small number of simple Device to Cloud scenarios; none of them could be improved by our approach.

Setup: Construction and analysis of IPCGs For the analysis, we constructed an IPCG for each integration scenario following the workflow sketched in Fig. 6. The integration scenarios are stored as process models in a BPMN-like notation [40] (similar to Fig. 1).

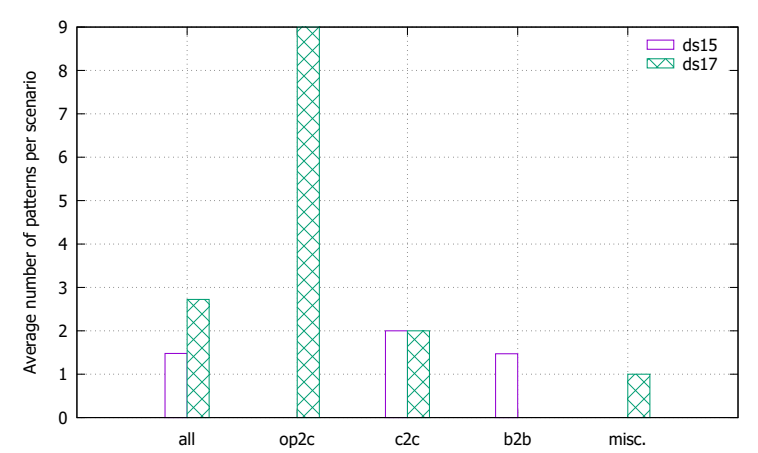

Figure 7: Pattern reduction per scenario.

The process models reference data specifications such as schemas (e.g., XSD, WSDL), mapping programs, selectors (e.g., XPath) and configuration files. For every pattern used in the process models, runtime statistics are available from benchmarks [38]. The data specifications are picked up from the 2015 content archive and from the current 2017 content catalog, while the runtime benchmarks are collected using the open-source integration system Apache Camel $[24]^{2}$ as used in SAP CPI. The mapping and schema information is automatically mined and added to the patterns as contracts, and the rest of the collected data as pattern characteristics. For each integration scenario and each optimization strategy, we determine if the strategy applies, and if so, if the cost is improved. This analysis runs in about two minutes in total for all 902 scenarios on our workstation.

We now discuss the improvements for the different kinds of optimization strategies identified in Sect. 2.

Improved Model Complexity: Process Simplification (OS-1). The relevant metric for the process simplification strategies from OS-1 is the model complexity, i.e. the average number of pattern reductions per scenario, shown in Fig. 7.

Results. Although all scenarios were implemented by integration experts, who are familiar with the modeling notation and the underlying runtime semantics, there is still a small amount of patterns per scenario that could be removed without changing the execution semantics. On average, the content reduction for the content from 2015 and 2017 was 1.47 and 2.72 patterns/IPCG, respectively, with significantly higher numbers in the OP2C domain.

Conclusions. (1) Even simple process simplifications are not always obvious to integration experts in scenarios represented in a controlflow-centric notation (e.g., current SAP CPI does not use BPMN Data Objects to visualize the data flow); and (2) the need for process simplification does not seem to diminish as integration experts gain more experience.

Improved Bandwidth: Data Reduction (OS-2). Data reduction impacts the overall bandwidth and message throughput [36]. To evaluate data reduction strategies from OS-2, we leverage the data element information attached to the IPCG contracts and characteristics, and follow their usages along edges in the graph, similar

\footnotetext{
${ }^{2}$ All measurements were conducted on a HP Z600 workstation, equipped with two Intel X5650 processors clocked at $2.67 \mathrm{GHz}$ with a 12 cores, $24 \mathrm{~GB}$ of main memory, running a 64-bit Windows 7 SP1 and a JDK version 1.7.0, with 2GB heap space.
} 


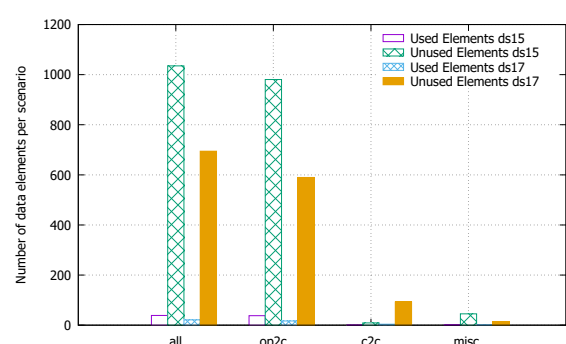

(a) Used vs. unused data elements

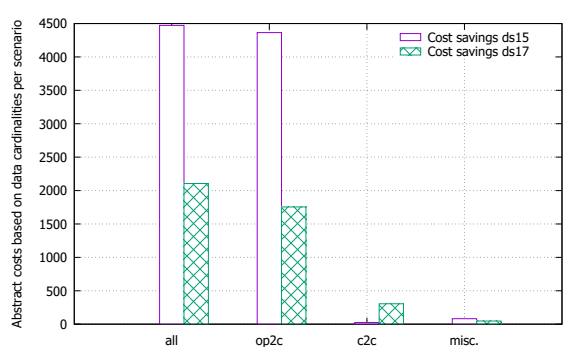

(b) Savings in abstract costs on unused data elements

Figure 8: Unused elements in integration scenarios.

to "ray tracing" algorithms [20]. We collect the data elements that are used or not used, where possible - we do not have sufficient design time data to do this for user defined functions or some of the message construction patterns, such as request-reply. Based on the resulting data element usages, we calculate two metrics: the comparison of used vs. unused elements in Fig. 8(a), and the savings in abstract costs on unused data elements in Fig. 8(b).

Results. There is a large amount of unused data elements per scenario for the OP2C scenarios; these are mainly web service communication and message mappings, for which most of the data flow can be reconstructed. This is because the predominantly used EDI and SOA interfaces (e.g., SAP IDOC, SOAP) for interoperable communication with on-premise applications define a large set of data structures and elements, which are not required by the cloud applications, and vice versa. In contrast, C2C scenarios are usually more complex, and mostly use user defined functions to transform data, which means that only a limited analysis of the data element usage is possible.

When calculating the abstract costs for the scenarios with unused fields, there is an immense cost reduction potential for the OP2C scenarios as shown in Fig. 8(b). This is achieved by adding a content filter to the beginning of the scenario, which removes unused fields. This results in a cost increase $\left|d_{i n}\right|=$ \#unused elements for the content filter, but reduces the cost of each subsequent pattern up to the point were the elements are used.

Conclusions. (3) Data flows can best be reconstructed when design time data based on interoperability standards is available; and (4) a high number of unused data elements per scenario indicates where bandwidth reductions are possible.

Improved Latency: Parallelization (OS-3). For the sequence-toparallel optimization strategies from OS-3, the relevant metric is the processing latency of the integration scenario. Because of the uncertainty in determining whether a parallelization optimization would be beneficial, we first report on the classification of parallelization candidates in Fig. 9(a). We then report both the improvements according to our cost model in Fig. 9(b), as well as the actual measured latency in Fig. 9(c).

Results. Based on the data element level, we classify scenario candidates as parallel, definitely non parallel, or potentially parallel in Fig. 9(a). The uncertainty is due to sparse information. From the 2015 catalog, $81 \%$ of the scenarios are classed as parallel, or potentially parallel, while the number for the 2017 catalog is $53 \%$. In both cases, the OP2C and B2B scenarios show the most improvement potential. Figure 9(b) shows the selection based on our cost model, which supports the pre-selection of all of these optimization candidates. The actual, average improvements per impacted scenario are shown in Fig. 9(c). The average improvements of up to 230 milliseconds per scenario must be understood in the context of the average runtime per scenario, which is 1.79 seconds. We make two observations: (a) the cost of the additional fork and join constructs in Java are high compared to those implemented in hardware [36], and the improvements could thus be even better, and (b) the length of the parallelized pattern sequence is usually short: on average 2.3 patterns in our scenario catalog.

Conclusions. (5) The parallelization requires low cost fork and join implementations; and (6) better runtime improvements might be achieved for scenarios with longer parallelizable pattern sequences.

\subsection{Case Studies}

We apply, analyze and discuss the proposed optimization strategies in the context of two case studies: the Replicate Material on-premise to cloud scenario from Fig. 1, as well as an SAP eDocument invoicing cloud to cloud scenario. These scenarios are part of the SAP CPI standard, and thus several users (i.e., SAP's customers) benefit immediately from improvements. For instance, we additionally implemented a content monitor pattern [37] that allowed analysis of the SAP CPI content. This showed the Material Replicate scenario was used by 546 distinct customers in 710 integration processes copied from the standard into their workspace - each one of these users is affected by the improvement.

Replicate Material (revisited). Recall from Sect. 1 that the Replicate Material scenario is concerned with enriching and translating messages coming from a CRM before passing them on to a Cloud for Customer service, as in Fig. 1. As already discussed, the content enricher and the message translator can be parallelized according to the sequence to parallel optimization from OS-3. The original and resulting IPCGs are shown in Fig. 2(a) and 2(b). No throughput optimizations apply.

Latency improvements. The application of this optimization can be considered, if the latency of the resulting parallelized process is smaller than the latency of the original process, i.e. if

$$
\begin{array}{r}
\operatorname{cost}(M C)+\max (\operatorname{cost}(C E), \operatorname{cost}(M T))+\operatorname{cost}(J R)+\operatorname{cost}(A G G) \\
<\operatorname{cost}(C E)+\operatorname{cost}(M T)
\end{array}
$$




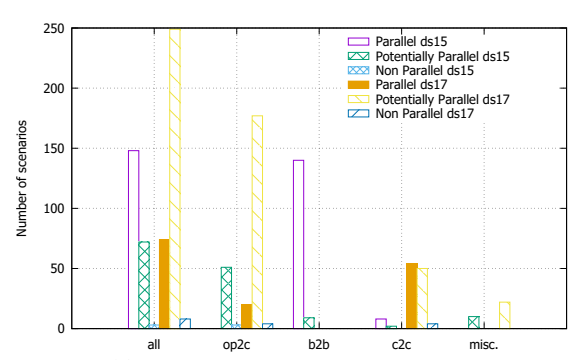

(a) Parallelization scenario candidates

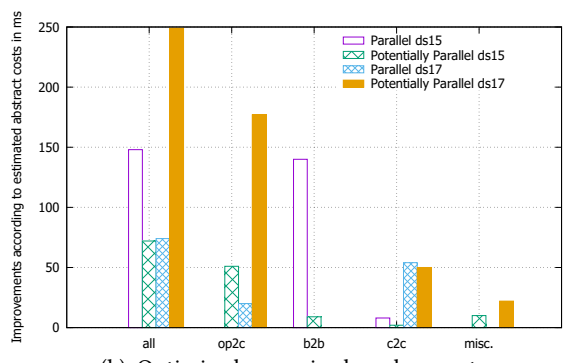

(b) Optimized scenarios based on costs

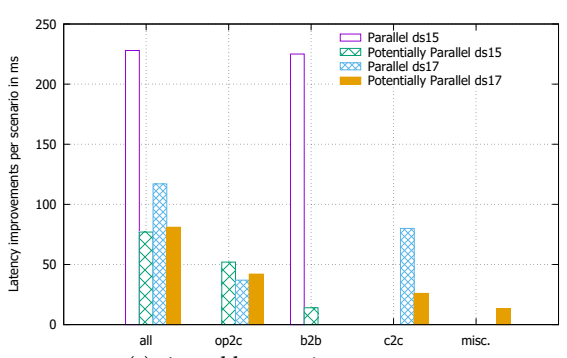

(c) Actual latency improvements

Figure 9: OS-3 "Sequence to parallel" optimization candidates on (a) integration flows, (b) optimization selection based on abstract cost model, and (3) actual latency improvements.

Subtracting $\max (\operatorname{cost}(C E), \cos t(M T))$ from both sides of the inequality, we are left with

$$
\operatorname{cost}(M C)+\operatorname{cost}(J R)+\cos t(A G G)<\min (\operatorname{cost}(C E), \cos t(M T))
$$

If we assume that the content enricher does not need to make an external call, its abstract cost becomes $\operatorname{cost}(C E)\left(\left|d_{i n}\right|,\left|d_{r}\right|\right)=\left|d_{i n}\right|$, and plugging in experimental values from a pattern benchmark [38], we arrive at the inequality (with latency costs in seconds)

$$
0.01+0.002+0.005 \nless \min (0.005,0.27)
$$

which tells us that the optimization is not beneficial in this case - the additional overhead is larger than the saving. However, if the content enricher does use a remote call, $\operatorname{cost}(C E)\left(\left|d_{i n}\right|,\left|d_{r}\right|\right)=$ $\left|d_{i n}\right|+\left|d_{r}\right|$, and the experimental values now say $\operatorname{cost}(C E)=0.021$. Hence the optimization is worthwhile, as

$$
0.01+0.002+0.005<\min (0.021,0.27) \text {. }
$$

Model Complexity. Following Sánchez-González et al. [42], we measure the model complexity as the node count. Hence, in this case, the optimization increases the complexity by three.

Conclusions. (7) The pattern characteristics are important when deciding if an optimization strategy should be applied (e.g., local vs. remote enrichment); and (8) there are goal conflicts between the different objectives, as illustrated by the trade-off between latency reduction and increasing model complexity.

eDocuments: Italy Invoicing. The Italian government accepts electronic invoices from companies, as long as they follow regulations - they have to be correctly formatted, signed, and not be sent in duplicate. Furthermore, these regulations are subject to change. This can lead to an ad-hoc integration process such as in Fig. 10 (simplified). Briefly, the companies' Fattura Electronica is used to generate a factorapa document with special header fields (e.g., Paese, IdCodice), then the message is signed and sent to the authorities, if it has not been sent previously. The multiple authorities respond with standard Coglienza, Risposta acknowledgments, that are transformed to a SendInvoiceResponse. We transformed the BPMN model to an IPCG, tried to apply optimizations, and created a BPMN model again from the optimized IPCG.

Our heuristics for deciding in which order to try to apply different strategies are "simplification before parallelization" and "structure before data", since this seems to enable the largest number of optimizations. Hence we first try to apply OS-1 strategies: the combine siblings rule matches the sibling Message Signers, since the preceding content-based router is a fork. (The signer is also side-effect free, so applying this rule will not lead to observably different behavior.) Next we try OS-3 strategies. Although heterogeneous parallelization matches for the $\mathrm{CE}$ and the Message Encoder, it is not applied since

$$
\operatorname{cost}(M C)+\cos t(J R)+\operatorname{cost}(A G G) \nless \min (\operatorname{cost}(C E), \operatorname{cost}(M E)),
$$

i.e., the overhead is too high, due to the low-latency, local CE. Finally, the early-filter strategy from OS-2 is applied for the Content Filter, inserting it between the Content Enricher and the Message Encoder. No further strategies can be applied. The resulting integration process translated back from IPCTG to BPMN is shown in Fig. 11. Conclusions. (9) The application order OS-1, OS-3, OS-2 seems most beneficial ("simplification before parallelization", "structure before data"); (10) an automatic translation from IPCGs to concepts like BPMN could be beneficial for connecting with existing solutions.

\section{RELATED WORK}

We presented related optimization techniques in Sect. 2. We now briefly situate our work within the context of other formalizations, beyond the already discussed BPMN [40] and PN [17] approaches. Enterprise Application Integration. Similar to the BPMN and PN notations, several domain-specific languages (DSLs) have been developed that describe integration scenarios. Apart from the EIP icon notation [23], there is also the Java-based Apache Camel DSL [24], and the UML-based Guaraná DSL [18]. However, none of these languages aim to be optimization-friendly formal integration scenario representations. Conversely, we do not strive to build another integration DSL. Instead we claim that all of the integration scenarios expressed in such languages can be formally represented in our formalism, so that optimizations can be determined that can be used to rewrite the scenarios.

There is work on formal representations of integration patterns, e.g. Mederly et al. [29] represents messages as first-order formulas and patterns as operations that add and delete formulas, and then applies AI planning to find an process with a minimal number of components. While this approach shares the model complexity objective, our approach applies to a broader set of objectives and optimization strategies. For the verification of service-oriented manufacturing systems, Mendes et al. [30] uses "high-level" Petri 


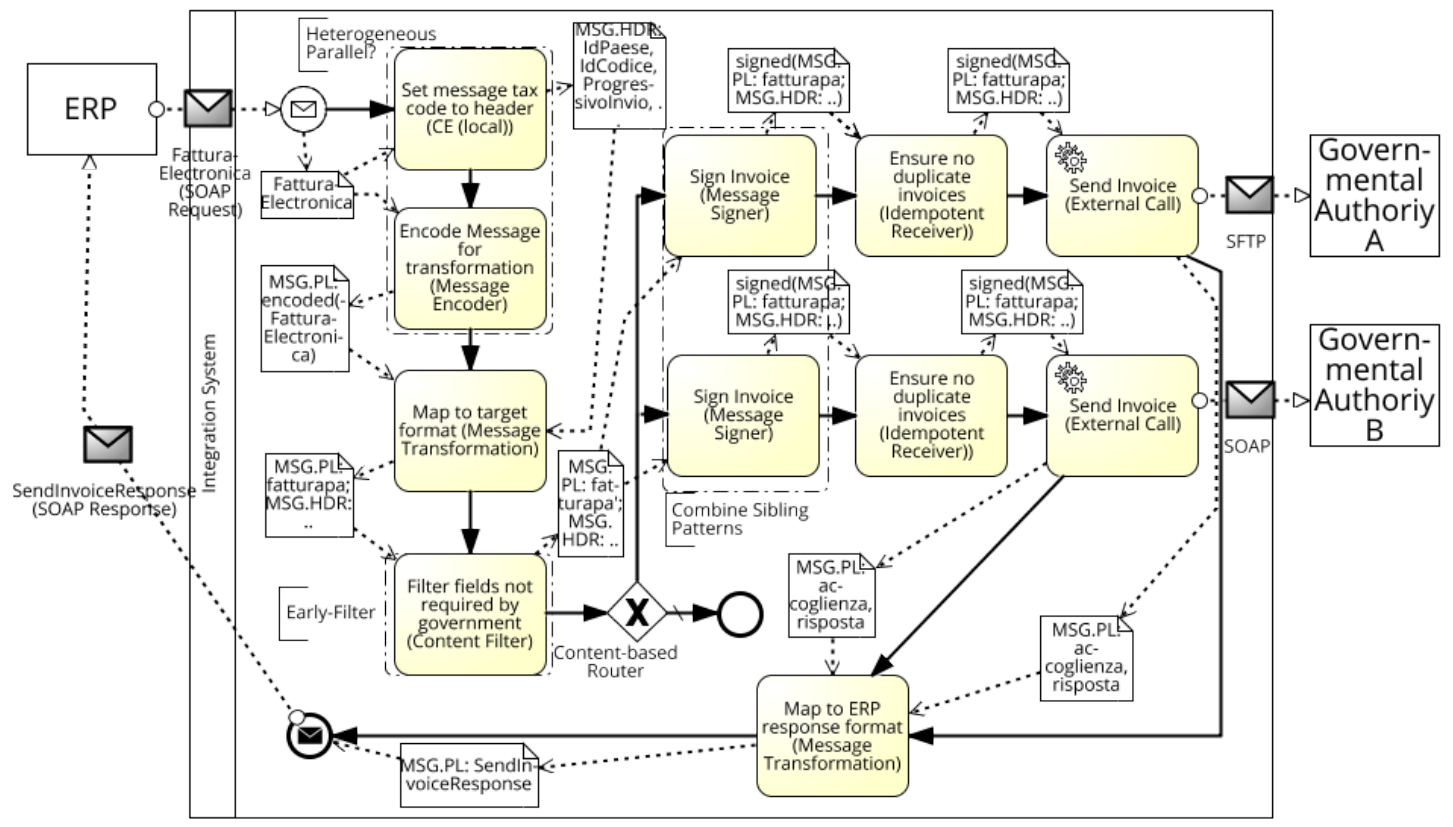

Figure 10: Country-specific invoicing (potential improvements as BPMN Group.)

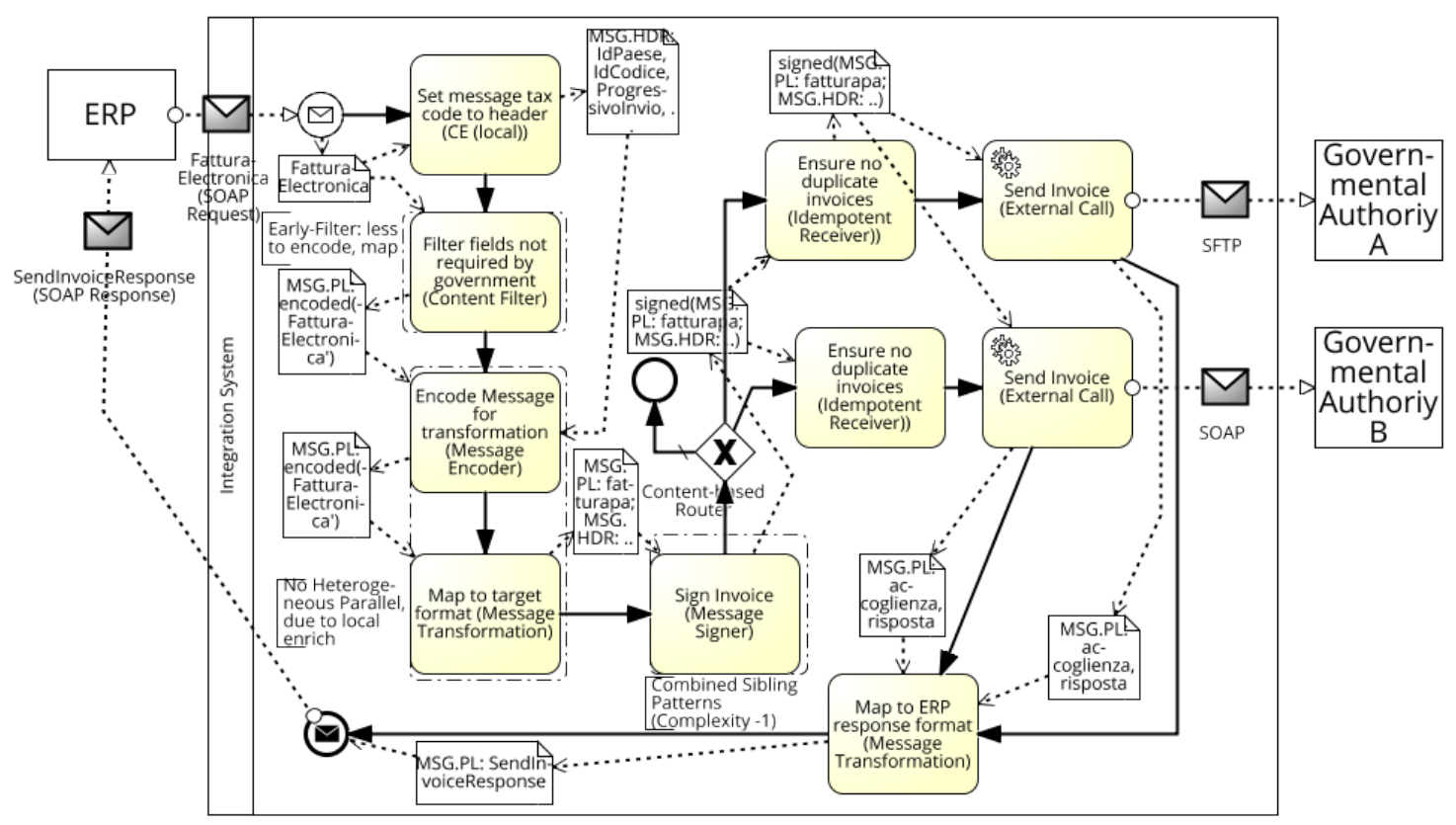

Figure 11: Invoice processing from Fig. 10 after application of strategies OS-1-3.

nets as a language instead of integration patterns, similar to the approach of Fahland and Gierds [17].

Business Process Management. Early algorithmic work by Sadiq and Orlowska [41] applied reduction rules to workflow graphs for the visual identification of structural conflicts (e.g., deadlocks) in business processes. Compared to process control graphs, we use a similar base representation, which we extend by pattern characteristics and data contracts. Furthermore, we use graph rewriting for optimization purposes. In Cabanillas et al. [13], the structural aspects are extended by a data-centered view of the process that 
allows to analyze the life cycle of an object, and check data compliance rules. Although this adds a view on the required data, it does not propose optimizations for the extended EIPs. The main focus is rather on the object life cycle analysis of the process.

\section{CONCLUSIONS}

This work addresses an important shortcoming in EAI research, namely the lack of optimization strategies, and the informality of descriptions of pattern compositions and optimizations (cf. RQ1-RQ3). We approached the questions by compiling a catalog of optimization strategies from the literature. We then developed a formalization of pattern compositions in order to precisely define optimizations, which we evaluated on data sets containing in total over 900 real world integration scenarios, and two brief case studies. We conclude that formalization and optimizations are relevant even for experienced integration experts (conclusions 1-2), with interesting choices (conclusions 3-4, 6), implementation details (conclusions 5, 10) and trade-offs (conclusions 7-9).

In further work, we plan to incorporate dynamic aspects into the formalization of patterns, for a more precise cost semantics. In addition, purely data related techniques like message indexing, fork path re-ordering and merging of conditions can be analyzed for their effects. Finally, multi-objective optimizations and heuristics for graph rewriting on the process level have to be further studied.

\section{ACKNOWLEDGMENTS}

We thank Jonas Kandels for implementation support, and the anonymous reviewers for suggestions and comments.

\section{REFERENCES}

[1] S. Abiteboul, M. Arenas, P. Barceló, M. Bienvenu, D. Calvanese, C. David, R. Hull, E. Hüllermeier, B. Kimelfeld, L. Libkin, W. Martens, T. Milo, F. Murlak, F. Neven, M. Ortiz, T. Schwentick, J. Stoyanovich, J. Su, D. Suciu, V. Vianu, and K. Yi. Research directions for principles of data management (abridged). SIGMOD Record, 45(4):5-17, 2017.

[2] K. Agrawal, A. Benoit, L. Magnan, and Y. Robert. Scheduling algorithms for linear workflow optimization. In IPDPS, pages 1-12, 2010.

[3] S. G. Ahmad, C. S. Liew, M. M. Rafique, E. U. Munir, and S. U. Khan. Dataintensive workflow optimization based on application task graph partitioning in heterogeneous computing systems. In IEEE BdCloud, pages 129-136, 2014.

[4] F. E. Allen. Control flow analysis. SIGPLAN Notices, 5(7):1-19, 1970.

[5] D. Battré, S. Ewen, F. Hueske, O. Kao, V. Markl, and D. Warneke. Nephele/ PACTs: a programming model and execution framework for web-scale analytical processing. In SoCC, pages 119-130, 2010.

[6] A. Benoit, M. Coqblin, J.-M. Nicod, L. Philippe, and V. Rehn-Sonigo. Throughput optimization for pipeline workflow scheduling with setup times. In Euro-Par Workshops, pages 57-67, 2012

[7] L. F. Bittencourt and E. R. M. Madeira. Hcoc: a cost optimization algorithm for workflow scheduling in hybrid clouds. fournal of Internet Services and Applications, 2(3):207-227, 2011.

[8] A. Böhm. Building Scalable, Distributed Applications with Declarative Messaging. $\mathrm{PhD}$ thesis, University of Mannheim, 2010.

[9] A. Böhm and C. Kanne. Demaq/transscale: Automated distribution and scalability for declarative applications. Information Systems, 36(3):565-578, 2011.

[10] M. Böhm, D. Habich, W. Lehner, and U. Wloka. Systemübergreifende Kostennormalisierung für Integrationsprozesse. In $B T W$, pages 67-86, 2009.

[11] M. Böhm, D. Habich, S. Preissler, W. Lehner, and U. Wloka. Cost-based vectorization of instance-based integration processes. Information Systems, 36(1):3-29, 2011.

[12] M. Böhm, U. Wloka, D. Habich, and W. Lehner. Model-driven generation and optimization of complex integration processes. In ICEIS (1), pages 131-136, 2008

[13] C. Cabanillas, M. Resinas, A. Ruiz-Cortés, and A. Awad. Automatic generation of a data-centered view of business processes. In CAiSE, pages 352-366. Springer, 2011.

[14] H. Ehrig, K. Ehrig, U. Prange, and G. Taentzer. Fundamentals of Algebraic Graph Transformation. Monographs in Theoretical Computer Science. Springer, 2006.
[15] H. Ehrig, M. Pfender, and H. J. Schneider. Graph-grammars: An algebraic approach. In Switching and Automata Theory, pages 167-180, 1973.

[16] D. Eyers, A. Gal, H.-A. Jacobsen, and M. Weidlich. Integrating Process-Oriented and Event-Based Systems (Dagstuhl Seminar 16341). Dagstuhl Reports, 6(8):21-64, 2017.

[17] D. Fahland and C. Gierds. Analyzing and completing middleware designs for enterprise integration using coloured petri nets. In CAiSE, pages 400-416, 2013.

[18] R. Z. Frantz, A. M. Reina Quintero, and R. Corchuelo. A domain-specific language to design enterprise application integration solutions. International fournal of Cooperative Information Systems, 20(02):143-176, 2011.

[19] J. R. Getta. Static optimization of data integration plans in global information systems. In ICEIS, pages 141-150, 2011.

[20] A. S. Glassner. An introduction to ray tracing. Elsevier, 1989.

[21] A. Habel and D. Plump. Relabelling in graph transformation. In ICGT, volume 2505, pages 135-147. Springer, 2002.

[22] I. Habib, A. Anjum, R. Mcclatchey, and O. Rana. Adapting scientific workflow structures using multi-objective optimization strategies. TAAS, 8(1):4, 2013.

[23] G. Hohpe and B. Woolf. Enterprise integration patterns: Designing, building, and deploying messaging solutions. Addison-Wesley, 2004.

[24] C. Ibsen and J. Anstey. Camel in Action. Manning, 2010.

[25] A. Kissinger, A. Merry, and M. Soloviev. Pattern graph rewrite systems. In DCM, pages 54-66, 2012.

[26] B. Kitchenham. Procedures for performing systematic reviews. Keele, UK, Keele University, 33(2004):1-26, 2004.

[27] G. Kougka and A. Gounaris. Optimization of data-intensive flows: Is it needed? Is it solved? In DOLAP, pages 95-98. ACM, 2014.

[28] G. Kougka, A. Gounaris, and A. Simitsis. The many faces of data-centric workflow optimization: A survey. CoRR, abs/1701.07723, 2017.

[29] P. Mederly, M. Lekavỳ, M. Závodský, and P. Navra. Construction of messagingbased enterprise integration solutions using AI planning. In CEE-SET, pages $16-29,2009$

[30] J. M. Mendes, P. Leitão, A. W. Colombo, and F. Restivo. High-level petri nets for the process description and control in service-oriented manufacturing systems. International fournal of Production Research, 50(6):1650-1665, 2012.

[31] F. Niedermann, S. Radeschütz, and B. Mitschang. Business process optimization using formalized patterns. BIS, 2011.

[32] F. Niedermann and H. Schwarz. Deep business optimization: Making business process optimization theory work in practice. In Enterprise, Business-Process and Information Systems Modeling, pages 88-102. Springer, 2011.

[33] K. Peffers, T. Tuunanen, M. A. Rothenberger, and S. Chatterjee. A design science research methodology for information systems research. $7 M I S, 24(3): 45-77,2007$.

[34] D. Plump and A. Habel. Graph unification and matching. In TAGT, pages 75-88, 1994.

[35] D. Ritter. Database processes for application integration. In BICOD, pages 49-61, 2017.

[36] D. Ritter, J. Dann, N. May, and S. Rinderle-Ma. Hardware accelerated application integration processing: Industry paper. In ACM DEBS, pages 215-226, 2017.

[37] D. Ritter, N. May, and S. Rinderle-Ma. Patterns for emerging application integration scenarios: A survey. Information Systems, 67:36 - 57, 2017.

[38] D. Ritter, N. May, K. Sachs, and S. Rinderle-Ma. Benchmarking integration pattern implementations. In ACM DEBS, pages 125-136, 2016.

[39] D. Ritter and S. Rinderle-Ma. Toward application integration with multimedia data. In IEEE EDOC, pages 103-112, 2017.

[40] D. Ritter and J. Sosulski. Exception handling in message-based integration systems and modeling using BPMN. Int. 7. Cooperative Inf. Syst, 25(2):1-38, 2016.

[41] W. Sadiq and M. E. Orlowska. Analyzing process models using graph reduction techniques. Information systems, 25(2):117-134, 2000.

[42] L. Sánchez-González, F. García, J. Mendling, F. Ruiz, and M. Piattini. Prediction of business process model quality based on structural metrics. In $E R$, pages $458-463$, 2010.

[43] T. Tirapat, O. Udomkasemsub, X. Li, and T. Achalakul. Cost optimization for scientific workflow execution on cloud computing. In ICPADS, pages 663-668, 2013.

[44] K. Vergidis, A. Tiwari, and B. Majeed. Business process analysis and optimization: Beyond reengineering. IEEE Transactions on SMC, Part C, 38(1):69-82, 2008.

[45] M. Vrhovnik, H. Schwarz, O. Suhre, B. Mitschang, V. Markl, A. Maier, and T. Kraft. An approach to optimize data processing in business processes. In $V L D B$, pages 615-626, 2007.

[46] P. Zhang, Y. Han, Z. Zhao, and G. Wang. Cost optimization of cloud-based data integration system. In WISA, pages 183-188, 2012. 